\title{
Reflets
}

Revue ontaroise d'intervention sociale et communautaire

\section{Pratiques quotidiennes des intervernantes et des intervenants dans la santé mentale auprès des enfants}

\section{Michel-André Beauvolsk et Jean-Marc Bélanger}

Volume 7, numéro 1, printemps 2001

Santé mentale et les défis de l'an 2001

URI : https://id.erudit.org/iderudit/026339ar

DOI : https://doi.org/10.7202/026339ar

Aller au sommaire du numéro

Éditeur(s)

Reflets : Revue ontaroise d'intervention sociale et communautaire

ISSN

1203-4576 (imprimé)

1712-8498 (numérique)

Découvrir la revue

Citer cet article

Beauvolsk, M.-A. \& Bélanger, J.-M. (2001). Pratiques quotidiennes des intervernantes et des intervenants dans la santé mentale auprès des enfants. Reflets, 7(1), 114-128. https://doi.org/10.7202/026339ar

Tous droits réservés (C) Reflets : Revue ontaroise d'intervention sociale et communautaire, 2001
Ce document est protégé par la loi sur le droit d'auteur. L'utilisation des services d'Érudit (y compris la reproduction) est assujettie à sa politique d'utilisation que vous pouvez consulter en ligne.

https://apropos.erudit.org/fr/usagers/politique-dutilisation/ 


\section{Pratiques quotidiennes des intervernantes et des intervenants dans la santé mentale auprès des enfants ${ }^{1}$}

Michel-André Beauvolsk et Jean-Marc Bélanger

Université Laurentienne

\section{Introduction}

\section{Encadrement théorique}

L'encadrement pour ces entrevues se base sur les recherches de Assay et Lambert (1999); Bachelor et Horvath (1999); Hubble, Duncan et Miller (1999); et Duncan et Miller (2000), qui ont fait des recherches sur le résultat de la thérapie auprès de la population 
cliente. En résumé, ces chercheurs identifient quatre facteurs de changement contribuant à un résultat positif chez la clientèle, sans égard à l'orientation théorique ou à la discipline professionnelle du thérapeute. Ces facteurs sont décrits ci-dessous en incluant leur contribution en pourcentage.

La première catégorie de ces quatre facteurs se nomme facteurs clients. Il s'agit du message «non actualisé» du dysfonctionnement familial. Les clientes et les clients sont souvent décrits comme les porteurs de messages du dysfonctionnement de la famille, les manufacturiers de la résistance et, dans les traditions les plus thérapeutiques, elles et ils sont les cibles pour les techniques d'intervention qu'on présume être les plus importantes. La cliente ou le client est rarement perçu dans le rôle de l'agent chef du changement. Tallman et Bohart (1999) présentent clairement le fait que la cliente ou le client est actuellement le contributeur le plus puissant du résultat de la psychothérapie avec les ressources qu'elle ou il amène dans la salle de thérapie et les influences sur sa vie en provenance de l'extérieur. Ainsi la persistance, l'ouverture, la confiance, l'optimisme, une grand-mère très supportante, ou un membre d'une communauté religieuse, par exemple, peuvent tous être des facteurs opérants dans la vie de l'individu, avant même que ce dernier entre en thérapie. Selon Assay et Lambert (1999), les facteurs clients comptent pour $40 \%$ de l'amélioration durant la thérapie.

Les facteurs relationnels sont la deuxième catégorie de facteurs. Bachelor et Horvath (1999) ont argumenté de façon convaincante que, suite à ce que la personne cliente apporte en thérapie, ses perceptions de la relation thérapeutique sont responsables pour la majorité des gains acquis en thérapie. Ces chercheurs ont élaboré un concept qui élargit la relation thérapeutique et qu'ils ont surnommé «l'alliance». L'alliance met l'accent sur la collaboration et encourage un partenariat entre la personne cliente et la personne thérapeute, afin d'atteindre les buts de la cliente ou du client (Bordin 1979). Les évaluations positives de cet esprit de collaboration des personnes clientes sont les meilleures prédictions de l'atteinte des buts des clients. On attribue un pourcentage de 
$30 \%$ aux facteurs relationnels comme responsables du taux de succès pour la thérapie.

La troisième catégorie de facteurs est appelée les facteurs de l'espérance et des attentes du système client. Cette classe de facteurs fait référence à la portion de l'amélioration qui dérive de la connaissance par la cliente ou le client d'être traité, de l'instillation de l'espérance et de la crédibilité perçue par le client du rationnel et des techniques de la thérapie. On attribue un pourcentage de $15 \%$ de résultats positifs à cette catégorie de facteurs.

Finalement, comme dernière catégorie de facteurs de changement, nous retrouvons les facteurs des modèles et des techniques d'intervention. Ces facteurs sont les croyances et les procédures uniques aux traitements spécifiques. Par exemple, songeons à certaines conditions telles la question miracle en thérapie orientée vers la solution, la désensibilisation en thérapie béhaviorale et les interprétations de transférence en thérapie freudienne. Ces modèles et techniques amènent des manières alternatives de conceptualiser et de conduire la thérapie lorsqu'un progrès ne semble pas venir. Les facteurs des modèles et des techniques contribuent un taux de $15 \%$ au changement en thérapie. Nous reviendrons à ces catégories dans la conclusion.

\section{But des entrevues}

Le but principal de nos entrevues avec les deux équipes de cliniciennes et de cliniciens du Centre de l'enfant et de la famille était basé sur l'hypothèse que le système-client possède les ressources nécessaires pour résoudre ou pour adresser la situation ou le problème présenté. À l'aide de notre encadrement théorique, nous avons posé des questions portant sur les quatre catégories de facteurs de changement. Nous vous présentons les résultats de ces entrevues selon l'expérience de l'équipe du Centre de l'enfant et de la famille. 


\section{Facteurs de changement}

\section{La clientèle}

La première question cernait la clientèle de l'équipe du Centre de l'enfant et de la famille. L'équipe a d'abord mentionné les effets de la restructuration des services en santé mentale sur le travail de tous les jours. Cette restructuration a entraîné la priorisation d'une population à servir. Historiquement, ce service s'adressait à une population volontaire aux besoins universels. Suite à cette restructuration, le Centre accorde la priorité à des populations avec des problématiques particulières et, à cause de cela, de plus en plus de demandes sont souvent refusées ou mises en attente. La population qui a la priorité est celle des familles avec des enfants qui ont des difficultés émotionnelles et qui ne sont pas capables de fonctionner dans les écoles.

La restructuration nous impose une priorisation de la population qui doit être servie... Avec cette restructuration, il y a des populations avec des caractéristiques particulières. Beaucoup de demandes sont acheminées et sont soit refusées ou mises en attente sur la liste d'attente. La population prioritaire sont des enfants qui ont tellement de difficultés émotionnelles et qui ne sont pas capables de fonctionner dans les lieux du foyer ou de l'école. Je crois que quand le Ministère a imposé ces critères là, qu'il a sous-estimé le montant de la population qui souffrait de ces mêmes problèmes là... Depuis que nous avons commencé à prioriser cette population là, ce sont des dossiers de la catégorie essentielle que nous gérons uniquement. Les dossiers qui tombent dans la catégorie investissement sont les listes d'attente. Certains d'entre eux attendent depuis plus d'un an parce que nous n'avons pas les ressources nécessaires pour accommoder les besoins de la population essentielle et les besoins de la population d'investissement. 
Lorsque le Ministère a ciblé cette population, il n'a sûrement pas pensé au nombre grandissant de ces familles. Depuis que le Centre a commencé à accorder la priorité à ces familles, les dossiers de la catégorie essentielle sont gérés en premier et le reste de la clientèle doit attendre. Les intervenantes remarquent aussi qu'elles voient de plus en plus de jeunes couples. Leur intervention apporte de l'assistance aux parents pour qu'ils puissent gérer les problèmes de leurs enfants. Auparavant, les intervenants dans le bien-être des enfants adressaient la relation parents-enfants. La différence présente semble résider dans le fait que les parents amènent les enfants à l'agence et les y abandonnent pour que celle-ci traite du problème et rééduque les enfants.

La philosophie souvent, c'est que les parents arrivent et [nous disent] «fix my kid». Quand ils disent «fix my kid», c'est une mentalité. Dans le fond, on nous «dump» la responsabilité de l'enfant.

Le gouvernement, dans cette affaire, a son propre agenda. Il a imposé son propre système de gérance des dossiers avec un système d'évaluation. Une intervenante le présente ainsi :

Il [le gouvernement] veut rendre nos services plus efficaces pour nous rendre plus efficaces, alors ils sont en train d'imposer des systèmes de gérance au dossier et des systèmes d'évaluation à l'entrée du dossier parce que tout le monde, on reçoit et évalue les dossiers pour être consistant à travers la province. C'est une grosse recherche et on doit prouver qu'on est efficace...

En effet une recherche provinciale est en cours sur les résultats face aux problèmes contenus dans ces dossiers pour juger de l'efficacité des services des agences.

Les intervenantes et les intervenants semblent être passés d'un rôle d'intervention à un rôle de gérance des besoins cliniques. Puisque les intervenants sont obligés de développer des liens de communication avec leurs partenaires, ils ont aussi développé une philosophie de clinique de gérance des dossiers. Voici comment 
une participante a exprimé cet ajustement dans son rôle d'intervenante.

Alors, et puis c'est pas juste aussi d'avoir un système de gérance administrative, c'est aussi d'avoir une philosophie clinique dans notre gérance de dossier. Ça c'est un rôle qui n'est pas toujours compris par nos partenaires, et puis c'est quelque chose qu'on doit se débattre pour maintenir. Alors on a eu beaucoup de formation dans le «wrap around" [qui] est une approche efficace mais aussi qui prend beaucoup de temps parce que tu peux avoir un partenaire qui influence un client d'une certaine façon qui va défaire ton plan d'intervention que tu as travaillé dessus pendant des mois. Alors c'est très important d'être capable de contrôler les influences dans le système familial et puis, [de] les amener à la table de façon consistante où tout le monde est sur la même page. C'est beaucoup de travail. Je ne sais pas, mais si jamais on comptait la proportion de temps passé en intervention individuelle familiale avec celle passée en collaboration avec nos partenaires, notre temps est peut-être plus passé à communiquer et à négocier avec nos partenaires.

Nous remarquons, cependant, qu'après avoir reçu une formation dans le modèle du "Wrap Around», les intervenantes sont plus en mesure de contrôler les influences dans le système familial, puis d'amener les membres de la famille à établir des façons d'agir consistantes où tout le monde est sur une même longueur d'onde, et ce, malgré les problématiques reliées aux échanges avec les partenaires pertinents.

\section{Les contraintes budgétaires}

Les contraintes budgétaires du gouvernement ont durement frappé les services du Centre de l'enfant et de la famille. Son budget a connu une baisse de près de $20 \%$, en plus de subir des restructurations, y compris la perte de deux sites. Puisque cet argent est 
octroyé selon la base de la population, les effets dans le nord de la province sont beaucoup plus désastreux puisque la proportion est beaucoup plus petite; conséquemment, les services coûtent plus cher à administrer. Occasionnellement, quelques rares nouveaux fonds sont parfois donnés pour accroître les services. Il semble qu'on s'attendait à ce que les intervenantes soient disponibles vingt-quatre heures sur vingt-quatre, et à ce qu'elles en fassent plus avec les mêmes salaires. Toutefois la réalité du problème du manque de ressources est plus profonde comme le manifeste cette intervenante.

Nous autres (les Centres de santé mentale pour enfants), on a été le plus frappé au niveau des budgets par le gouvernement. Je pense qu'on a perdu 25 à $30 \%$ de notre budget pendant le règne de ce gouvernement là. On a subi des restructurations, on a perdu des sites. Ça a affecté notre structure administrative. Depuis ce temps-là l'argent n'a pas été retourné. Quand on regarde le budget total pour les Centres de santé mentale pour toute la province, ça égale le budget d'un hôpital enseignant en Ontario. Alors on est vraiment un petit réseau quand on se compare à des gros réseaux tels la santé et l'éducation pour les services à l'enfance. On sent vraiment que le gouvernement ne nous compare pas bien.

Les contraintes budgétaires ont fait plus que de restreindre des services spécifiques, elles contribuent aussi à un déséquilibre au sein de la structure globale.

\section{La restructuration dans les écoles}

La restructuration de l'éducation dans les écoles a affecté les services en santé mentale pour enfants. Les demandes en temps et en services auprès des enseignantes et enseignants dans nos écoles ont beaucoup augmenté. Les conseillères et les conseillers pédagogiques ont presque tous disparu. Lorsqu'un jeune est en crise, il est souvent immédiatement suspendu. Les autorités scolaires 
ont beaucoup moins de tolérance qu'auparavant pour les enfantsproblèmes. En conséquence, les intervenantes du Centre de l'enfant et de la famille travaillent avec beaucoup plus d'enfants agressifs qui défient l'autorité, qui ont un langage très grossier et qui, physiquement, peuvent poser des gestes dangereux. Il n'est pas réaliste de renvoyer ces enfants à la maison parce que les parents travaillent souvent tous les deux; demander que l'un des deux reste à la maison n'est pas une option, puisque le salaire des parents est requis pour nourrir ces enfants. On observe que les parents d'aujourd'hui semblent avoir beaucoup moins de ressources pour faire face à ces problèmes. Une intervenante offre le bilan suivant de la restructuration du système scolaire au niveau du secondaire.

Lorsqu'on a fermé nos huit centres, surtout au secondaire, on a fait un suivi un an après pour savoir où étaient nos élèves de l'ancienne section dix-neuf. De ce que [nous] avons su, à peu près un tiers était toujours à l'école et avait reçu des services réorganisés $d u$ Conseil, et ils réussissaient à fonctionner comme cela. Un autre tiers était à l'école mais avait beaucoup de difficultés avec des suspensions de tous types. Puis un autre tiers avait disparu, ayant décroché ou étant en prison, ou vivait sur la rue.

On peut donc voir que la demande de services de santé mentale dans les écoles semble augmenter et non décroître. Le problème est que plusieurs jeunes adolescents tombent dans les "craques» du système et au lieu de solutionner leurs problèmes à l'école même, on les renvoie sans se préoccuper de qui leur adviendra, ce qui ne fait que déplacer le problème. Il y a des attentes que ceci se fera au niveau de la cellule familiale. Toutefois, dans le cas de familles pauvres ou dépendantes de l'aide sociale, la flexibilité de ces parents de prendre en main un comportement acrimonieux devient encore plus mince. Les contraintes qui leur sont imposées sont tellement incongrues qu'elles deviennent elles-mêmes oppressives. 


\section{Les besoins}

L'équipe du Centre de l'enfant et de la famille s'est aussi penchée sur les besoins créés par les changements sociaux. De prime abord, les membres de l'équipe constatent qu'un changement au sein du système entraîne d'autres changements au sein du suprasystème, parce que plusieurs autres ressources, qui étaient souvent disponibles dans d'autres secteurs, ne le sont plus maintenant.

Au niveau de la société il y a eu de grands changements au niveau des services municipaux, du bien-être, de l'éducation, etc. Et puis ça fait que cela a augmenté la demande sur les services de santé mentale et en même temps ça a enlevé beaucoup des supports sur lesquels on dépendait pour mieux servir les gens.

On remarque que cette restructuration affecte aussi le fonctionnement du travail dans lequel doit opérer l'équipe. La demande étant à la hausse, il y a beaucoup moins de temps pour une supervision clinique adéquate.

On a besoin de plus de direction clinique, mais c'est pas la faute de X... parce qu'elle est débordée, mais considérant la quantité des dossiers que l'on a... des fois on est pris, on n'est pas certain...

Les exigences du travail pour suffire à la demande, tant bien que mal, se font donc au détriment de la supervision clinique, ce qui fait que les intervenantes doivent développer de plus en plus d'habiletés de survie au sein de leur travail. Non seulement doiton se questionner à savoir si les besoins de la clientèle sont satisfaits, mais aussi ceux des intervenantes dans leur encadrement professionnel.

\section{Un travail efficace}

Nous avons demandé aux intervenantes quels besoins devraient être, à leur avis, remplis pour que leur travail soit plus efficace. Naturellement plus de direction clinique a été identifiée comme 
un besoin puisqu'une certaine incertitude fait en sorte que la supervision clinique aiderait face aux choix à faire et aux décisions à prendre. Mais on fait aussi mention de certaines autres possibilités. Le fait d'avoir de plus grands locaux à l'école élémentaire donnerait plus de possibilités pour travailler avec les jeunes, particulièrement ceux âgés entre 3 à 8 ans. En fait, on considère qu'il faut établir une bonne relation avec le personnel enseignant et que pour ce faire il faut savoir «ajuster» sa pratique.

Beaucoup de notre réussite est basée sur notre relation avec les gens dans les écoles. Il faut que tu ailles audelà de ce que tu vas faire dans l'école. Il faut donner un coup de main avec quelque chose d'autre, les aider, puis tout d'un coup ils sont prêts [à travailler] à l'intégration d'un élève.

Les intervenantes ont aussi souhaité avoir la possibilité de développer de nouveaux programmes de prévention afin d'adresser et de prévenir certains comportements non désirables. Une intervenante considère que cela ne donne rien de faire des interventions après coup.

Ça donne rien de faire des interventions plus tard. Si tu pouvais intervenir plus tôt peut-être qu'il $y$ aurait moins de situations qui arriveraient... Une fois qu'ils nous arrivent le dommage est fait... On touche la surface tout le temps. Ça devient frustrant car on a déjà travaillé [dans le passé] d'une autre façon.

Pour être plus efficace dans leur travail les intervenantes et intervenants du Centre de l'enfant et de la famille croient qu'on doit faire plus de travail auprès des parents qui, à cause de diverses circonstances, deviennent de plus en plus détachés de leurs enfants. Une intervenante l'indique de la façon suivante :

On ne travaille pas nécessairement avec des ados venant de familles pauvres. Même parmi les familles professionnelles, les deux parents sont tellement submergés 
dans les demandes de leur travail qu'ils ne sont pas accessibles émotionnellement à leurs enfants. Alors ce sont des enfants qui s'élèvent par eux-mêmes.

Une autre intervenante fait cette affirmation :

Il $y$ a deux parents qui travaillent à plein temps et qui ont parfois deux ou trois jobs. Les parents ne sont pas accessibles, puis ils ont perdu le sens de c'est quoi leur rôle. Si tu es un bon parent il faut que tu fournisses tout ce qu'il faut sur le plan matériel. C'est quelque chose qui a été perdu un tout petit peu de savoir c'est quoi être un parent.

Il faudrait donc des ressources pour être capable de travailler plus étroitement avec les parents, mais là encore le facteur temps et les exigences de l'emploi présentent des obstacles, même si on réalise qu'une meilleure coordination aiderait à répondre aux besoins des familles qui éprouvent de la difficulté à être émotionnellement accessibles à leurs enfants. Mais il existe aussi une nouvelle catégorie de clients, soit les parents qui possèdent la garde partagée de leurs enfants. Pour ces parents, les protocoles ne sont pas bien établis. Comment revendiquer les droits de ces enfants dans ces circonstances?

Comme solution viable afin d'être le plus efficace possible, l'équipe utilise différentes approches. Entre autres, mentionnons le modèle du traitement du trauma intergénérationnel (InterGenerational Trauma Treatment Model) qui offre une approche englobant plusieurs a priori tels un aspect moral et une approche éducative. C'est une manière plus amicale et moins menaçante d'intervenir et qui vise la connexion des jeunes et de leurs parents, car à la base de certains problèmes de conduite on retrouve un désordre d'attachement. Le travail se fait sur la relation enfantparents. C'est un modèle qui travaille sur la base d'éléments positifs. L'équipe utilise aussi le modèle narratif de White et Epston (1990) qui utilisent les histoires familiales pour retrouver un sens à la vie et trouver un encadrement utile et positif face au problème vécu. 


\section{Espoir et commentaires}

Pour conclure l'entrevue, nous avons invité les intervenantes et les intervenants à nous faire part de ce qui les motive à continuer de travailler dans un domaine aussi stressant. Voici certaines de leurs réponses.

Moi, quand je travaille avec les clients, je suis toute pleine d'énergie.

Il faut que tu aimes les enfants et les familles pour travailler dans ce domaine. Tu ne devrais pas être ici si t'aimes pas ça. Vraiment!

C'est excitant quand tu vois les changements à travers ton travail.

Toutefois, on sent une certaine inquiétude face à la clientèle.

Moi,j'ai des craintes pour les familles. Comme les familles n'ont pas de services qu'est-ce qui va leur arriver? La région manque de services. Alors je ne sais pas.

L'un des désirs manifesté par l'équipe, cependant, est celui d'établir une meilleure relation et communication avec les autres agences impliquées dans la gérance des mêmes cas.

Une des conséquences de la réforme dans d'autres communautés a été l'intégration de différents services, agences et de centres communautaires en une agence unique avec la Société de l'aide à l'enfance. Les centres indépendants sont alors disparus et puis nous sentons que c'est un danger pour les familles qui veulent accéder à des services qui sont maintenant en voie de disparition. Cette centralisation amène une perception d'un manque de confidentialité ou de confiance, de sorte que les droits de confidentialité des familles seraient plus à risques. Alors, ici à Sudbury, il y a vraiment une volonté des agences d'avoir des partenariats. C'est un peu la Coop que l'on vit ici; c'est une mesure que l'amalgamation 
n'était pas perçue comme la réponse dans la réorganisation des services aux enfants.

Suite à tous ces témoignages, on est en droit de soulever certaines questions. Les services cliniques de santé mentale seraientils autonomes sous la tutelle de la Société de l'aide à l'enfance? Quelle ouverture ou accessibilité le grand public aurait-il aux services cliniques sous la tutelle de la Société de l'aide à l'enfance? Les services de santé mentale deviendraient-ils secondaires? Enfin, quel sort est réservé aux agences de services de santé mentale si elles deviennent amalgamées en un seul service dans un plus grand réseau?

\section{Conclusion}

Ce qui ressort de l'entrevue peut se résumer à deux valeurs professionnelles fondamentales : la créativité et l'efficacité. Ce que les participantes de l'entrevue ont clairement décrit reflète la complexité de leur travail et leur manque de ressources. Face aux facteurs théoriques décrits, le vécu des intervenantes, tel que présenté, semble démontrer que les facteurs relationnels prennent une place primordiale dans leur travail de tous les jours. Malgré les réalités impondérables auxquelles elles font face, les travailleuses sociales misent sur la possibilité d'établir une alliance, une coopération pro-active entre elles-mêmes et les personnes qu'elles servent. En travaillant de pair avec leurs clients, elles peuvent davantage établir des buts réalistes avec leur clientèle et les atteindre.

Les facteurs clients viennent en second lieu. Les clients proviennent de milieux souvent difficiles au chapitre des ressources parentales, financières et familiales. Puisque les clients sont les agents de changement les plus puissants dans ces interactions, il importe pour les travailleuses sociales d'établir une connexion avec leurs clients afin d'utiliser ces énergies de changement. La persistance, l'ouverture et l'optimisme des clients vont faciliter l'atteinte des buts fixés avec les clients. 
Les facteurs de l'espérance et des attentes du système client soustendent aussi le travail accompli. Les clients font appel au professionnalisme des travailleuses sociales dans l'espérance qu'elles puissent les aider à améliorer leur vie en général à travers la modification de certains comportements.

Les facteurs du modèle et des techniques d'intervention semblent entrer dans la considération la plus large des services offerts et disponibles. Les clients croient que les techniques et les méthodes les plus appropriées dans la livraison des services seront utilisées même si ces croyances sont rarement verbalisées.

Ce qui ressort de ces entrevues indique clairement quelles sont les difficultés du travail à accomplir, les manques de ressources, les politiques mouvantes et la variété de partenaires d'intervention. Mais ce qui ressort encore plus fort consiste en l'engagement, l'enthousiasme et l'amour des enfants que ces travailleuses sociales partagent dans l'accomplissement de leur travail. Elles croient vraiment dans la santé mentale des enfants.

Ça me donne satisfaction le travail que je fais.

Il faut que tu aimes les enfants et les familles pour travailler dans ce domaine.

\section{Références}

Assay,T.P., et Lambert, M.J. (1999). The empirical case for the common factors in therapy: Quantitative findings. In M. A. Hubble, B. L. Duncan, et S. D. Miller (Eds.), The heart and soul of change: What works in therapy (pp. 33-56). Washington, DC : APA Press.

Bachelor, A., et Horvath, A. (1999). The therapeutic relationship. In M. A. Hubble, B. L. Duncan, et S. D. Miller (Eds.), The heart and soul of change: What works in therapy (pp. 133-178). Washington, DC : APA Press.

Bordin, E. S . (1979). The generalizability of the psychoanalytic concept of the working alliance. Psychotherapy: Theory, Research and Practice, 16, 252-260.

Duncan, L., et Miller, S. D. (2000). The Heroic Client. Doing Client-Directed Outcome-Informed Therapy. San-Francisco : Jossey-Bass.

Hubble, M. A., Duncan, B. L., et Miller, S. D. (1999). Directing attention to what works. In M. A. Hubble, B. L. Duncan, et S. D. Miller (Eds.), The heart and soul of change:What works in therapy (pp. 407-448). Washington, DC : APA Press. 
Lambert, M.J. (1992). Implications of outcome research for psychotherapy integration. In C. Norcross et M. R. Goldfried (Eds.), Handbook of psychotherapy integration (pp. 94-129). New York : Basic.

Tallman, K., et Bohart, A. (1999).The client as a common factor: Clients as selfhealers. In M. Hubble, B. Duncan, et S. D. Miller, (Eds.), The heart and soul of change: What works in therapy (pp. 91-132). Washington, DC : APA Press.

White, M., et Epston, D. (1990). Narrative means to a therapeutic end. New York : Norton.

\section{Note}

1. Ont participé aux entrevues : Linda Dugas, Michelle Forest, Lucie Gélinas, Lynn Prévost, Carole Gauthier-Salewski, Jeannine Rouselle, Marc Trottier et Michelle Bernier-Wilson. 